
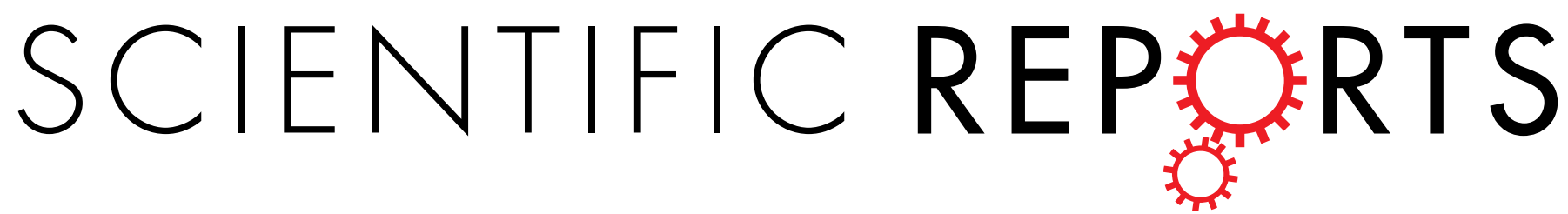

\title{
OPEN The rubber tree genome shows expansion of gene family associated with rubber biosynthesis
}

Received: 08 January 2016

Accepted: 06 June 2016

Published: 24 June 2016

\section{Nyok-Sean Lau,1,2, Yuko Makita², Mika Kawashima², Todd D. Taylor ${ }^{3}$, Shinji Kondo 4 , Ahmad Sofiman Othman ${ }^{1,5}$, Alexander Chong Shu-Chien ${ }^{1,5}$ \& Minami Matsui ${ }^{2}$}

Hevea brasiliensis Muell. Arg, a member of the family Euphorbiaceae, is the sole natural resource exploited for commercial production of high-quality natural rubber. The properties of natural rubber latex are almost irreplaceable by synthetic counterparts for many industrial applications. A paucity of knowledge on the molecular mechanisms of rubber biosynthesis in high yield traits still persists. Here we report the comprehensive genome-wide analysis of the widely planted $H$. brasiliensis clone, RRIM 600. The genome was assembled based on $\sim 155$-fold combined coverage with Illumina and PacBio sequence data and has a total length of $1.55 \mathrm{~Gb}$ with $72.5 \%$ comprising repetitive DNA sequences. A total of 84,440 high-confidence protein-coding genes were predicted. Comparative genomic analysis revealed strong synteny between $\boldsymbol{H}$. brasiliensis and other Euphorbiaceae genomes. Our data suggest that $H$. brasiliensis's capacity to produce high levels of latex can be attributed to the expansion of rubber biosynthesis-related genes in its genome and the high expression of these genes in latex. Using cap analysis gene expression data, we illustrate the tissue-specific transcription profiles of rubber biosynthesis-related genes, revealing alternative means of transcriptional regulation. Our study adds to the understanding of $H$. brasiliensis biology and provides valuable genomic resources for future agronomic-related improvement of the rubber tree.

Hevea brasiliensis Muell. Arg, the Pará rubber tree, or most commonly known as the rubber tree, is a medium to large monoecious and cross-pollinated tropical tree belonging to the family Euphorbiaceae (spurge) ${ }^{1}$. This family of flowering plants encompasses more than 7,500 species spread across 300 genera of herbs, shrubs and trees. Besides H. brasiliensis, Euphorbiaceae includes a number of plants with economic importance, such as cassava (Manihot esculenta), the castor oil plant (Ricinus communis) and Barbados nut (Jatropha curcas). The genus Hevea is comprised of eleven species ${ }^{2}$, namely H. brasiliensis, Hevea bethamiana, Hevea camporum, Hevea camargoana, Hevea guianensis, Hevea microphylla, Hevea nitida, Hevea pauciflora, Hevea paludosa, Hevea rigidifolia and Hevea spruceana ${ }^{3-5}$. All Hevea species are diploid $(2 n=2 \mathrm{x}=36)$ with the exception of triploid H. guianensis $(2 n=2 \mathrm{x}=54)$ and the existence of one genotype of $H$. pauciflora with $2 n=2 \mathrm{x}=18^{6,7}$. Although Hevea behaves as a diploid $^{8}$, it is proposed that Hevea has an amphidiploid origin stabilized during the course of evolution ${ }^{9}$. Among the genus members, only $H$. brasiliensis yields an economically viable amount of latex that makes up most (99\%) of the world's natural rubber production. In 2014, global natural rubber production was estimated at 12 million tons with a corresponding value of 22 billion US dollars (http://www.lgm.gov.my).

The rubber tree is indigenous to the Amazon basin in South America, but is now widely planted in Southeast Asia, especially in Malaysia, Indonesia and Thailand. The domestication of rubber trees outside Brazil occurred more than 100 years ago with the transfer of $H$. brasiliensis seedlings to Asia ${ }^{10}$. H. brasiliensis is a deciduous tree that grows to $30-40 \mathrm{~m}$ tall and can live up to 100 years in the wild ${ }^{11}$. Natural rubber is a high molecular weight polymer made of repeating isoprene units in the cis-configuration. Natural rubber differs from synthetic rubber in polymer length and side chain composition, which contribute to its superior performance properties in elasticity,

${ }^{1}$ Centre for Chemical Biology, Universiti Sains Malaysia, 11900 Bayan Lepas, Penang, Malaysia. ${ }^{2}$ Synthetic Genomics Research Group, RIKEN Center for Sustainable Resource Science, Biomass Engineering Research Division, Tsurumi, Yokohama, Kanagawa 230-0045, Japan. ${ }^{3}$ Laboratory for Integrated Bioinformatics, RIKEN Center for Integrative Medical Sciences, Tsurumi, Yokohama, Kanagawa 230-0045, Japan. ${ }^{4}$ Transdisciplinary Research Integration Center, National Institute of Polar Research, Tachikawa, Tokyo 190-8518, Japan. ${ }^{5}$ School of Biological Sciences, Universiti Sains Malaysia, 11800 Minden, Penang, Malaysia. Correspondence and requests for materials should be addressed to A.C.S.-C. (email: alex@usm.my) or M.M. (email: minami@riken.jp) 


\begin{tabular}{|l|c|}
\hline Feature & Value \\
\hline Number of scaffolds & 189,316 \\
\hline Longest scaffold (Kb) & 871.19 \\
\hline N50 scaffold length (Kb) & 67.24 \\
\hline Total size of scaffolds (Gb) & 1.55 \\
\hline Number of contigs & 262,709 \\
\hline N50 contig length (Kb) & 20.75 \\
\hline Longest contig (Kb) & 325.50 \\
\hline Total size of all contigs (Gb) & 1.51 \\
\hline GC content (\%) & 34.17 \\
\hline Number of predicted protein-coding gene models & 84,440 \\
\hline Average transcript length (bp) & 3,069 \\
\hline Average number of exons per gene & 4.97 \\
\hline Average coding sequence length (bp) & 971 \\
\hline Average exon length (bp) & 196 \\
\hline Average intron length (bp) & 478 \\
\hline
\end{tabular}

Table 1. Statistics of the $H$. brasiliensis genome assembly.

resilience and efficient heat dispersion. Hence, despite accounting for less than $50 \%$ of total rubber consumption, natural rubber is still indispensable for heavy-duty tires and high-performance engineering components.

In $H$. brasiliensis, rubber is synthesized on the surface of rubber particles suspended in the latex of laticifers or specialized parenchyma cells ${ }^{12}$. Six to seven years after planting, a rubber tree can be tapped for latex by controlled wounding of the bark tissue. H. brasiliensis clone RRIM 600, a secondary clone originally developed from clones Tijr 1 and PB 86, is the most widely planted clone in Malaysia and Thailand ${ }^{13,14}$. RRIM 600 is categorized as a medium yielding clone that produces $\sim 1,350 \mathrm{~kg}$ of latex per hectare per year in estates over 20 years old. This clone shows moderate tolerance to cold, drought and wind, but is susceptible to disease caused by the pathogenic fungal Phytophthora spp. (http://www.rubberboard.org.in).

Despite its economic importance, past attempts in genetic analyses to improve $H$. brasiliensis rubber production and other desirable traits have been progressing slowly. A major hurdle for crop improvement of $H$. brasiliensis is the lack of a comprehensive whole-genome overview and therefore, a holistic knowledge of the latex biosynthetic process. A previous attempt to assemble the $H$. brasiliensis genome produced a fragmented draft using 43 -fold sequence data ${ }^{14}$. A high-quality genome assembly for $\mathrm{H}$. brasiliensis is imperative for discovery of the precise pathways and genes responsible for desirable agronomic traits. Here, we report a more complete $H$. brasiliensis genome assembly that allows genomic comparison with other sequenced plant genomes and cap analysis gene expression (CAGE) studies on tissue-specific transcription of genes, particularly those related to rubber biosynthesis. Our effort unraveled genome-level insights on the evolutionary relationship of $H$. brasiliensis with other Euphorbiaceae species. Most importantly, our analysis reveals possible contributors to $H$. brasiliensis's capacity to produce high levels of latex and alternative means of transcriptional regulation for rubber biosynthesis.

\section{Results}

Genome sequencing and assembly. The genome assembly of $H$. brasiliensis was constructed using the whole-genome shotgun sequencing approach utilizing both Illumina and PacBio reads. A total of 288.84 Gb ( 134-fold coverage) of raw sequencing data was generated using the Illumina platform from both paired-end and mate-pair libraries with different insert sizes (Supplementary Table S1). The genome was assembled using Platanus, a de Brujin graph-based assembler ${ }^{15}$. The size of the genome has been estimated to be $2.15 \mathrm{~Gb}$ based on 17-mer depth distributions of Illumina paired-end reads, and this is consistent with a previous estimate ${ }^{16}$ (Supplementary Figure S1). The Platanus assembly was further improved using long PacBio reads through the implementation of the PBJelly2 software ${ }^{17}$. A $10-\mathrm{Kb}$ SMRTbell library was prepared and sequenced using 100 SMRT Cells, which yielded $45.25 \mathrm{~Gb}(\sim 21$-fold coverage) of sequence with a typical average read length of 6,852 bp (Supplementary Figure S2). The resulting final assembly spans $1.55 \mathrm{~Gb}$ with the largest scaffold being 871 $\mathrm{Kb}$ (Table 1). The genome assembly consists of 189,316 scaffolds with an N50 size of $67.2 \mathrm{~Kb}$. Using high coverage sequence data and the application of PacBio long reads, the N50 size of our H. brasiliensis genome assembly was increased 23-fold and the number of scaffolds was decreased three-fold compared to the previously published assembly ${ }^{14}$. Our current $H$. brasiliensis genome assembly represents a great improvement from the previous one, and allows for detailed and accurate functional annotation. The assembly was anchored to 18 linkage groups based on the published linkage map ${ }^{8}$ (Supplementary Figure S3). In total, 189 scaffolds could be anchored to the genetic map through restriction fragment length polymorphism (RFLP) markers. The GC content of the assembled $H$. brasiliensis genome is $34.17 \%$, similar to those of the sequenced genomes of R. communis $(32.5 \%)^{18}$, J. curcas $(33.7 \%)^{19}$ and M. esculenta $(34.86 \%)^{20}$ from the Euphorbiaceae family (Fig. 1).

The completeness of the assembly was evaluated by aligning EST sequences of $H$. brasiliensis to the genome assembly. Of the 51,631 H. brasiliensis ESTs available from NCBI, 96.8\% were detected in the assembly (Supplementary Table S2). The assembly was also validated by aligning assembled transcripts from RNA sequencing (RNA-seq) of different $H$. brasiliensis tissues ${ }^{14,21-24}$. More than $93.7 \%$ of the transcripts could be aligned to the 


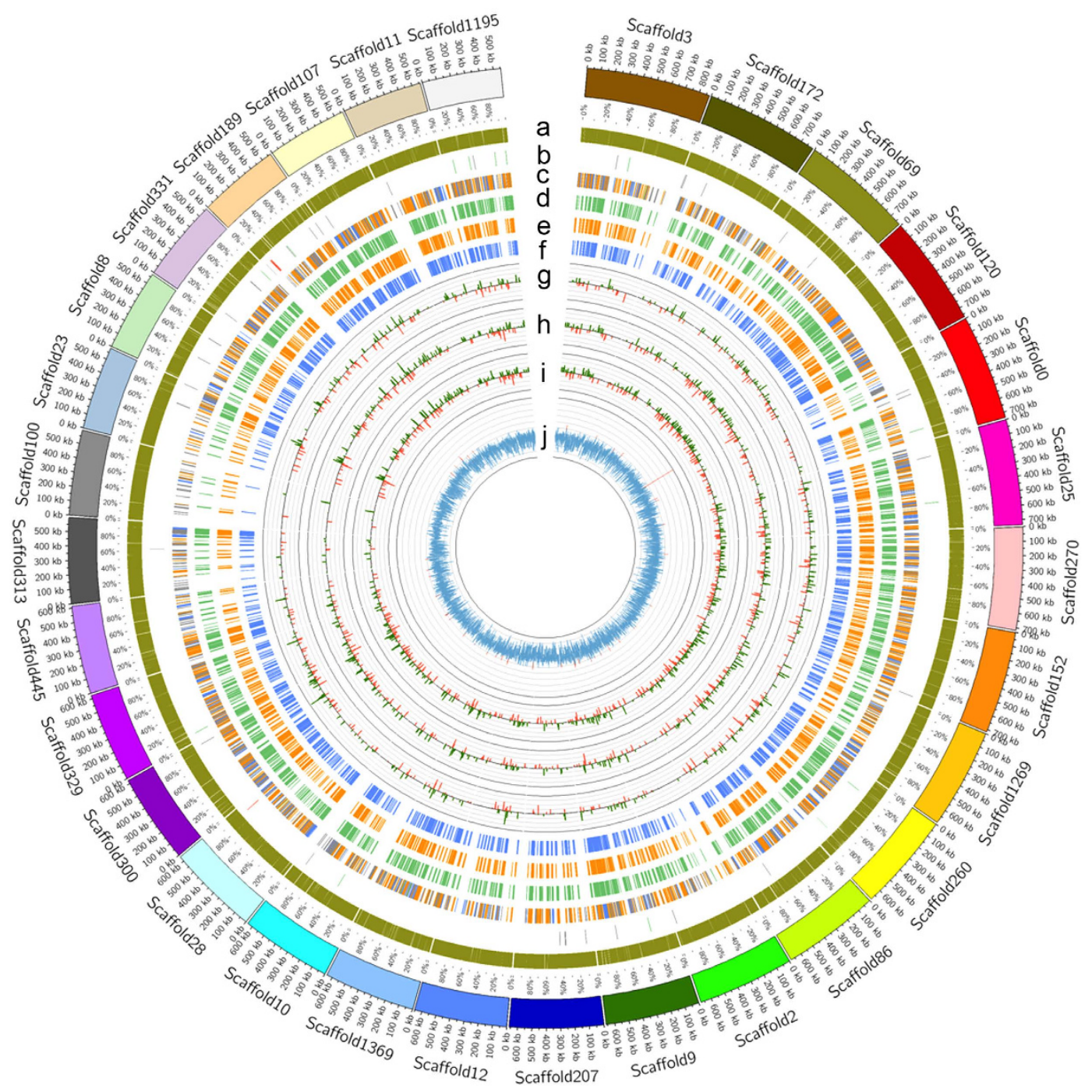

Figure 1. Characterization of the $\boldsymbol{H}$. brasiliensis genome. Circos plot of the 30 longest scaffolds. (a) Repeats; (b) Non-coding RNAs (rRNAs, tRNAs and other ncRNAs are represented by red, green and grey lines, respectively); (c) Gene model annotation against the NCBI non-redundant protein database (BLAST matches to R. communis, M. esculenta, J. curcas and other organisms are represented by blue, green, orange and grey lines, respectively); (d) Orthologous genes in M. esculenta; (e) Orthologous genes in J. curcas; (f) Orthologous genes in R. communis; (g) CAGE tags per million (TPM) in latex (TSSs mapped to the sense and antisense strand are represented in green and red, respectively); (h) CAGE TPM in leaf (TSSs mapped to the sense and antisense strands are represented in green and red, respectively); (i) CAGE TPM in bark (TSSs mapped to the sense and antisense strands are represented in green and red, respectively) (j) GC content (values of $>50 \%$ and $<=50 \%$ are represented in red and blue, respectively).

assembly, indicating near-complete coverage of the gene space in the assembly. We also detected a total of 445 and 416 mitochondrial and chloroplast DNA fragment insertions into the nuclear genome covering 594,389 and 230,702 bp, respectively (Supplementary Table S3). In flowering plants, the transfer of DNA from the mitochondria or plastids to the nucleus has been identified ${ }^{25}$. In the $H$. brasiliensis genome, these insertions include three large mtDNA fragments $(32-36 \mathrm{~Kb})$.

The extent of genome duplications in $H$. brasiliensis was analyzed by aligning $H$. brasiliensis scaffolds against itself using reciprocal BLAST matches. The 90 pairs of scaffolds that contained the largest number of paralogous gene pairs were selected and visualized in a dotplot (Supplementary Figure S4). Although genome triplications that were identified in the R. communis genome ${ }^{18}$ were not observed, this approach identified a total of 47 duplicated regions.

Repeat content of the $\boldsymbol{H}$. brasiliensis genome. Transposable elements represent major constituents of plant genomes. A combination of de novo and homologue-based approaches resulted in the identification of 1,783,541 repetitive sequences, accounting for $72.5 \%$ of the assembled genome (Table 2 ). This level of 


\begin{tabular}{|l|c|c|c|}
\hline Repeat elements & $\begin{array}{c}\text { Copies } \\
\text { (number) }\end{array}$ & $\begin{array}{c}\text { Length occupied } \\
\text { (bp) }\end{array}$ & $\begin{array}{c}\text { Genome } \\
(\%)\end{array}$ \\
\hline Retrotransposon & 881,720 & $888,699,356$ & 57.310 \\
\hline SINE & 367 & 43,121 & 0.003 \\
\hline LINE & 25,098 & $17,662,094$ & 1.139 \\
\hline LTR/Copia & 184,681 & $181,996,743$ & 11.737 \\
\hline LTR/Gypsy & 649,589 & $675,583,092$ & 43.567 \\
\hline LTR/others & 21,985 & $13,414,306$ & 0.865 \\
\hline DNA transposon & 48,232 & $18,463,525$ & 1.191 \\
\hline Unknown & 461,056 & $194,823,043$ & 12.564 \\
\hline Satellites & 234 & 34,261 & 0.002 \\
\hline Simple repeats & 326,501 & $18,738,351$ & 1.211 \\
\hline Low complexity & 65,798 & $3,632,769$ & 0.235 \\
\hline Total & $1,783,541$ & $1,124,391,305$ & 72.510 \\
\hline
\end{tabular}

Table 2. Repeat element analysis in the $H$. brasiliensis genome.

repetitiveness is similar to those of the tobacco $(72-79 \%)^{26}$, hot pepper $(76.4 \%)^{27}$ and cultivated cotton $(68.5 \%)^{28}$ genomes, but higher than those of castor bean $(50.33 \%)^{18}$ and cassava $(25.7-36.9 \%)^{20}$ from the Euphorbiaceae family (Supplementary Table S4). The noticeable proliferation of transposable elements in the H. brasiliensis genome has likely expanded its genome size compared to other Euphorbiaceae species. Overall, retrotransposons occupy the majority of the $H$. brasiliensis nuclear genome ( $57.3 \%)$, of which $\sim 11.7 \%$ and $\sim 43.6 \%$ are Copia and Gypsy elements, respectively. In contrast, DNA transposons only represent $\sim 1.2 \%$ of the nuclear DNA. Closer inspection revealed that the 3.71 ratio of Gypsy to Copia LTR-retrotransposons in the rubber genome is similar to that of the sorghum genome (3.66) ${ }^{29}$. The ratio of Gypsy to Copia elements in the genome generally varies between species $^{30}$.

Gene prediction and functional annotation. The Maker- $\mathrm{P}^{31,32}$ pipeline that combines ab initio gene predictions, homology-based searching and transcriptome alignments was used for genome annotation. Protein sequences from the M. esculenta, $R$. communis, J. curcas, P. trichorpca and Viridiplantae datasets from Swiss-Prot were used as homology-based evidence. Assembled contigs of RNA-seq reads from leaf, latex and bark tissues ${ }^{21}$ were used as transcript evidence. Only gene predictions that are supported by protein or transcript evidence are included in the final gene models. A total of 84,440 protein-coding genes were predicted, with an average transcript length of $3,069 \mathrm{bp}$, an average coding length of $971 \mathrm{bp}$ and an average of 4.97 exons per gene. Among the predicted genes, $76.5 \%$ of the encoded proteins showed homology to sequences in the NCBI non-redundant protein database (Supplementary Table S5). Additionally, 39.7\%, 74.7\%, 56.6\% and $72.8 \%$ of the genes could be functionally annotated using the Swiss-Prot, TrEMBL, GO and KEGG databases, respectively. GO annotation revealed that the $H$. brasiliensis genome maintains more genes associated with 'binding' and 'catalytic activity' subcategories than other genomes (Supplementary Figure S5). Furthermore, at least one conserved domain was detected in $65.5 \%$ of the predicted protein sequences by comparison against the InterPro database. In addition to protein-coding genes, non-coding RNAs in the assembly were also identified (Supplementary Table S6). A total of 739 transfer RNAs (tRNAs), 271 ribosomal RNAs (rRNAs), 192 spliceosomal RNAs, 231 small nucleolar RNAs and 206 micro RNAs were identified in the H. brasiliensis genome assembly.

Comparative analyses between rubber tree and other plants. The H. brasiliensis genome contains similar patterns of orthologous gene sets in comparison to representative species from monocotyledons (Oryza sativa) and dicotyledons (Arabidopsis thaliana, Vitis vinifera and Solanum lycopersicum), sharing a total of 8,627 common gene families (Fig. 2a). Proteome comparison between H. brasiliensis and other species from Euphorbiaceae (R.communis, M. esculenta and J. curcas) revealed that a large cluster of 12,406 gene families is shared among the four Euphorbiaceae genomes (Fig. 2b). The 1,785 gene families that are unique to $\mathrm{H}$. brasiliensis are significantly enriched with genes related to molecular function categories such as 'zinc ion binding', 'cation binding' and 'transition metal ion binding' (Supplementary Table S7). Of the Euphorbiaceae species with sequenced genomes, $M$. esculenta is the closest relative of $H$. brasiliensis. Consecutively, the largest number of gene clusters from 14,930 gene families is shared between $H$. brasiliensis and M. esculenta compared to the other plants examined in this study. Ks plots show that H. brasiliensis and M. esculenta diverged later than the divergence of R. communis and J. curcas in the Euphorbiaceae family (Fig. 2c). The macro-synteny between the genome of $H$. brasiliensis and those of distantly related species (R. communis, M. esculenta, J. curcas), as well as the more distantly diverged species (A. thaliana), was evaluated (Fig. 3). We found strong synteny between $H$. brasiliensis and other Euphorbiaceae species, but much weaker synteny between $H$. brasiliensis and A. thaliana. Alignment of $H$. brasiliensis scaffolds to their best-matched M. esculenta, R. communis, J.curcas and A. thaliana genomic regions was performed to analyze intergenome collinearity between these genomes (Fig. 4). On average, $\mathrm{H}$. brasiliensis gene blocks showed one-to-one, one-to-two and one-to-three synteny relationships with their orthologues in J. curcas, M. esculenta and R. communis, respectively. Comparison between H. brasiliensis and A. thaliana revealed that many regions of the $H$. brasiliensis genome have two, but some have three or more, homologous regions in A. thaliana. 

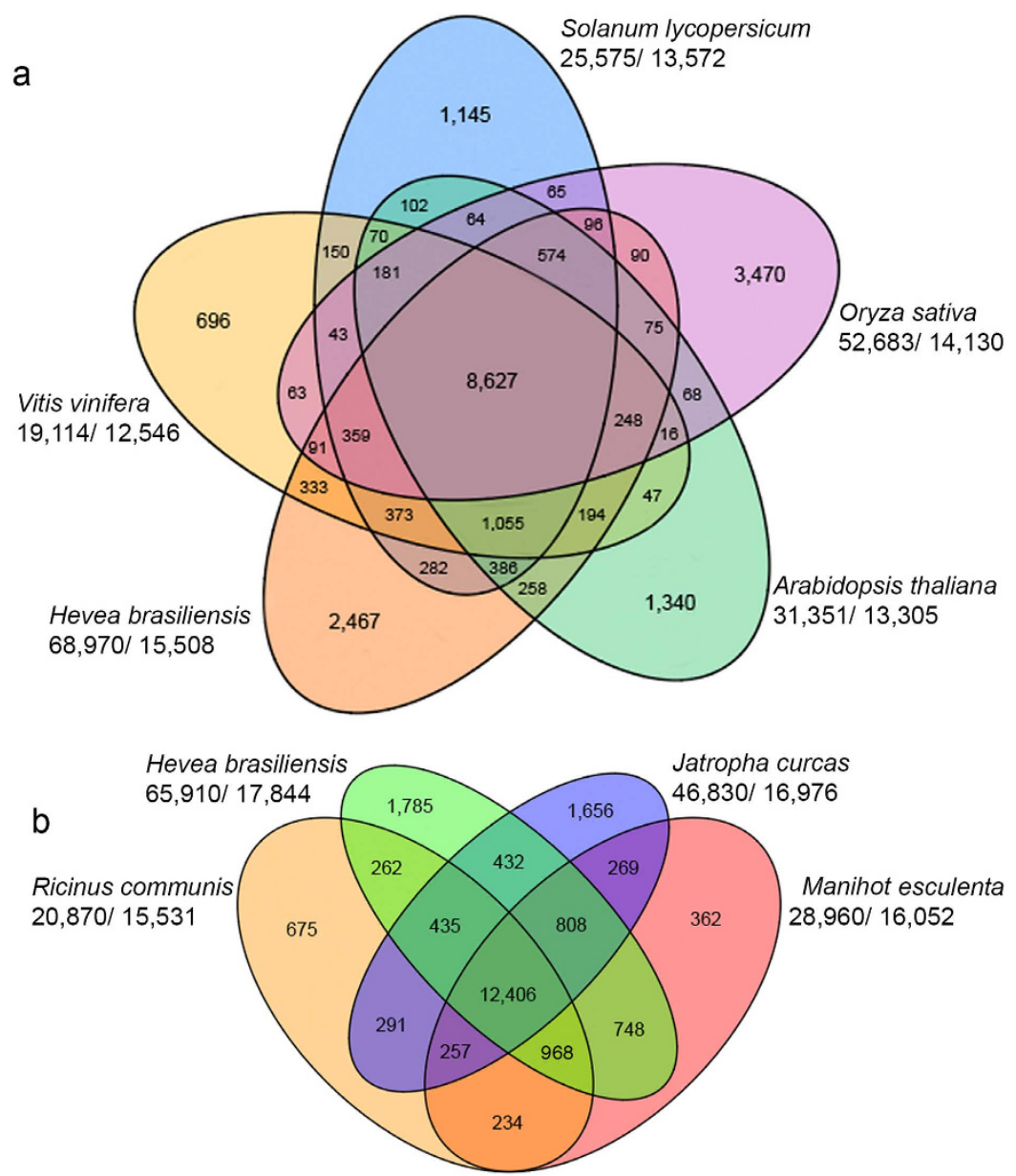

C

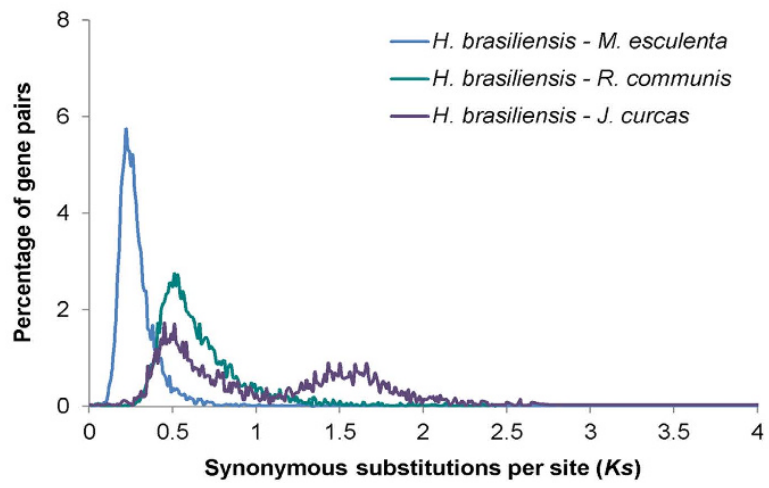

Figure 2. H. brasiliensis comparative genomics. Venn diagrams show unique and shared gene families between and among representative (a) angiosperms and (b) Euphorbiaceae. The number of genes in the families and the number of gene families are indicated under each species name. (c) Distribution of synonymous nucleotide substitution $(K s)$ rates. The blue, turquoise and purple lines represent the $K s$ distribution of orthologous gene pairs in H. brasiliensis-M. esculenta, H. brasiliensis-R. communis and H. brasiliensis-J. curcas, respectively. 8,136, 7,399 and 3,456 single-copy orthologs, were used for the H. brasiliensis-M. esculenta, $H$. brasiliensis-R. communis and H. brasiliensis-J. curcas analyses, respectively.

Transcription factor families. Within the H. brasiliensis genome, 3,142 putative transcription factors were classified into 89 subfamilies (Supplementary Table S8). Despite having a larger genome size, the core set of transcription factors in $H$. brasiliensis does not significantly differ from other plants. $H$. brasiliensis showed a very similar distribution of transcription factor families to A. thaliana, S. lycopersicum, O. sativa and R. communis. In comparison with these other plants, $H$. brasiliensis and $O$. sativa both have three times more FAR1 transcription factors in their genomes. FAR1 is related to a mutator-like transposase and is involved in modulation of phyA-mediated light signaling in $A$. thaliana ${ }^{33}$. The high frequency of this family in the genome may indicate 

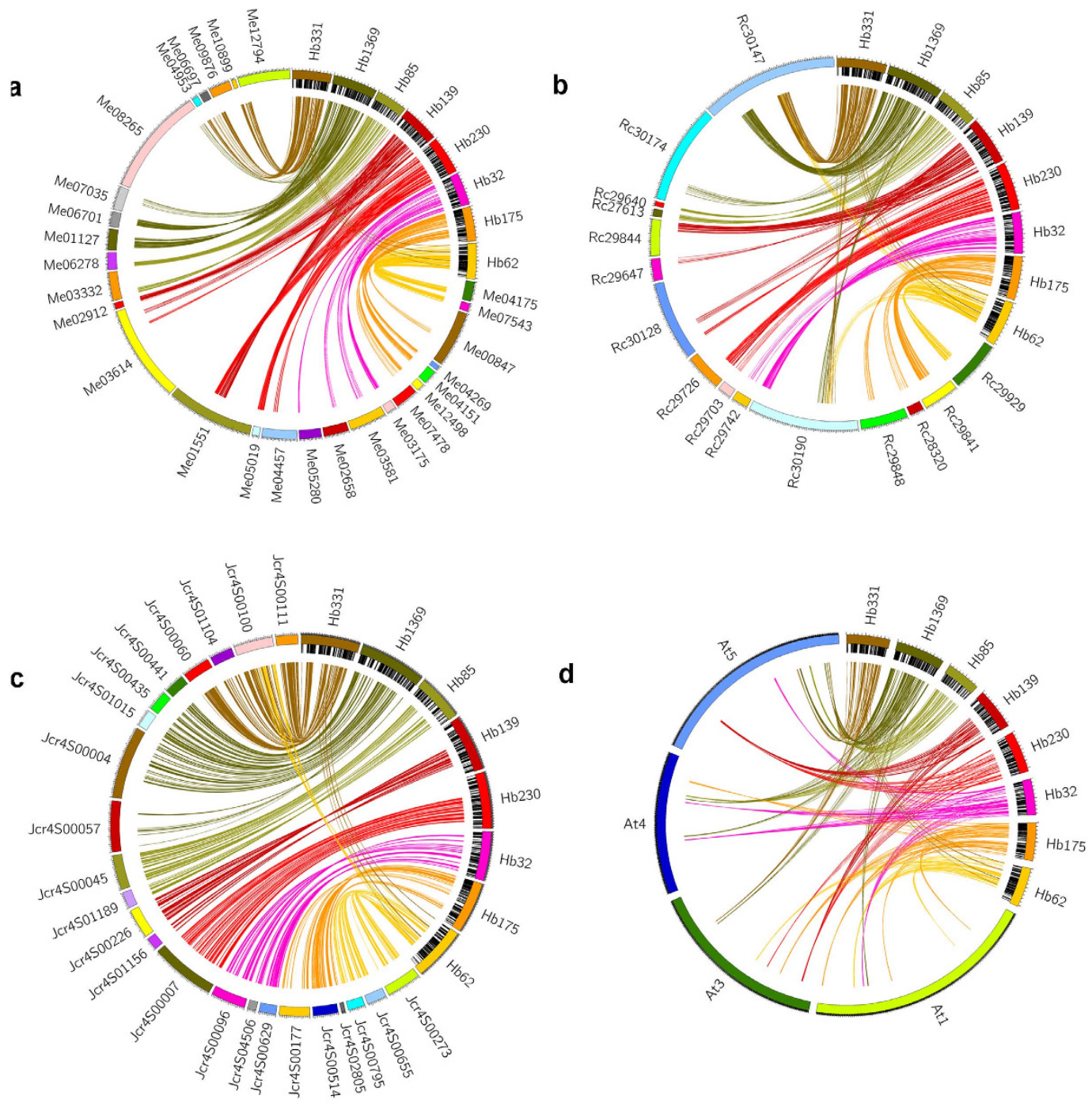

Figure 3. Macro-synteny between $H$. brasiliensis ( $\mathrm{Hb})$ and (a) M. esculenta (Me), (b) R. communis (Rc), (c) J. curcas (Jcr) and (d) A. thaliana (At). Labels around the circles correspond to scaffold numbers in $H$. brasiliensis, M. esculenta, R. communis, and J. curcas, and chromosome numbers in A. thaliana.

a more diverged role of this transcription factor in $H$. brasiliensis and O. sativa. Overall, MYB constitutes the most abundant group of transcription factors in $\mathrm{H}$. brasiliensis. These particular groups of transcription factors are important regulators of plant development, including hormone signal transduction, disease resistance and secondary metabolism ${ }^{34}$.

Disease resistance. Disease resistance is one of the most important traits identified as a target for rubber plant breeding. The rubber tree is susceptible to several fungal diseases, including South American leaf blight (Microcyclus ulei), abnormal leaf fall (Phytopthora spp.), powdery mildew (Oidium heveae), Corneyspora leaf fall (Cornyespora cassiicola), pink disease (Corticium salmonicolor) and white root disease (Rigidoporus spp. $)^{2}$. Genes related to disease resistance (R genes) play an important role in the resistance mechanism of plants, and information on these genes in the $H$. brasiliensis genome will provide a basis for improvement of disease resistance.

We identified 483 disease resistance-related genes, which is the highest among the four Euphorbiaceae plants examined here (Supplementary Table S9). This expansion of $\mathrm{R}$ genes in H. brasiliensis suggests an enhanced ability to detect and defend against pathogen invasion in latex-producing $H$. brasiliensis. These $\mathrm{R}$ genes found in the $H$. brasiliensis genome belong to the nucleotide-binding site (NBS) resistance genes and constitute about $0.57 \%$ of all $\mathrm{H}$. brasiliensis genes. The NBS-encoding genes can be classified into multiple subfamilies, including 186 NBS-leucine-rich repeat (NBS-LRR), 45 coiled-coil (CC)-NBS-LRR, 35 CC-NBS, 15 Toll interleukin-1 receptor-NBS-LRR (TIR-NBS-LRR) and 4 TIR-NBS. Some of these disease resistance-related genes were found organized in clusters in the $H$. brasiliensis genome. In particular, scaffold284, scaffold589 and scaffold2863 were found to contain clusters containing four, six and four disease resistance-related genes, respectively 
a
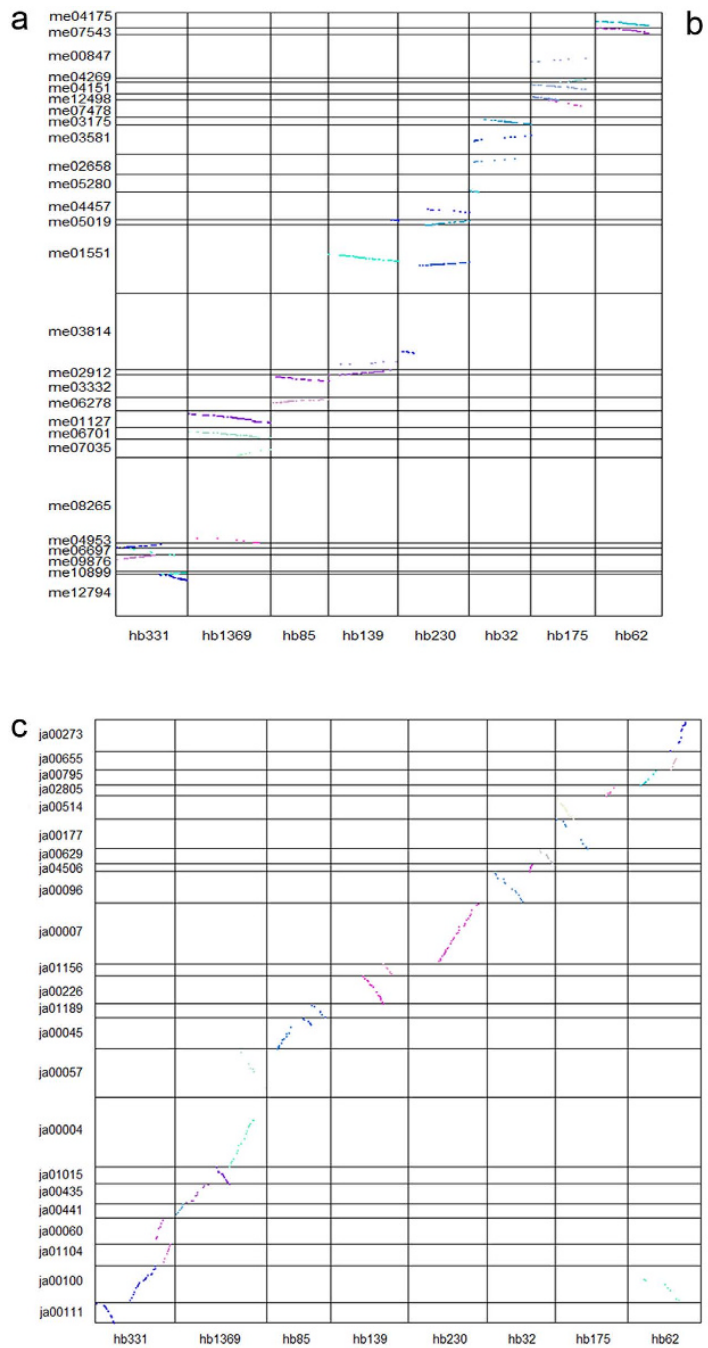

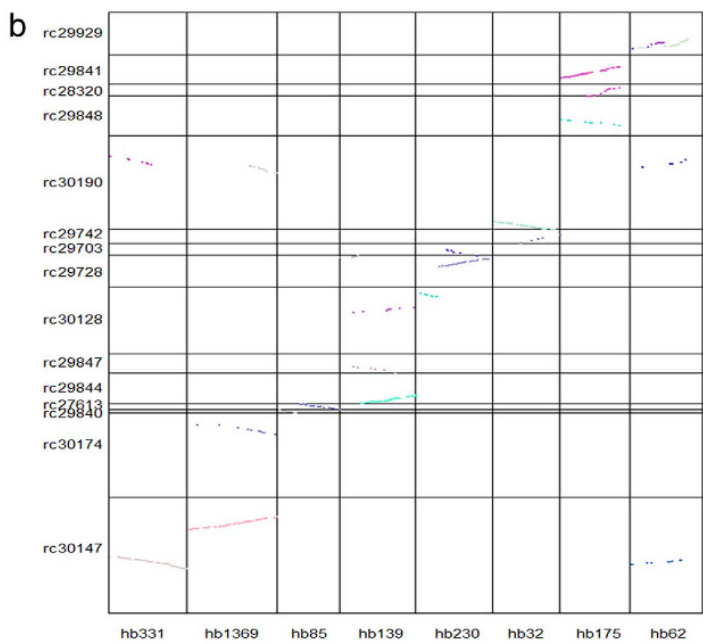

d

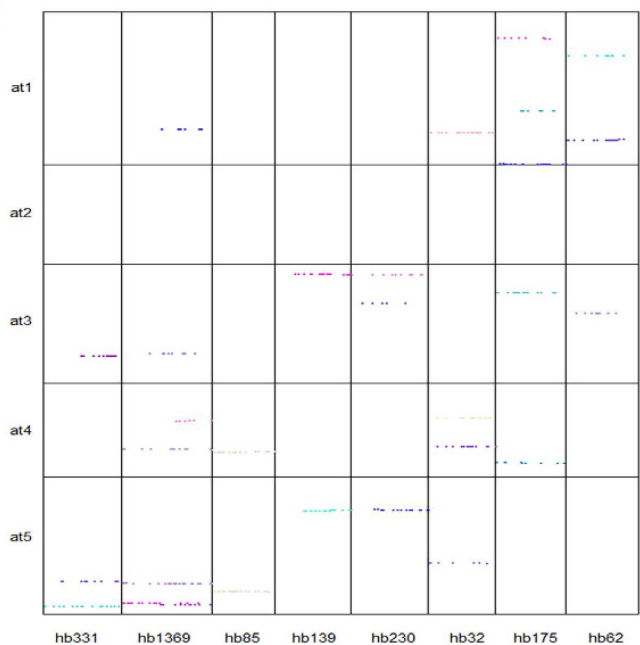

Figure 4. Dot-plot analyses between H. brasiliensis and (a) M. esculenta, (b) R. communis, (c) J. curcas scaffolds and (d) A. thaliana chromosomes. Each dot represents a collinear gene pair between $H$. brasiliensis, M. esculenta, R. communis, J. curcas and A. thaliana (BLASTP $e$-value cutoff $<10^{-5}$ ). Different coloured dots represent different collinear blocks. Eight scaffolds of $H$. brasiliensis that have more than 15 homologous genes in M. esculenta, R. communis, J. curcas, and A. thaliana are shown.

(Supplementary Figure S6). These observations are consistent with previous classical genetic and molecular data which reports that plant disease resistance genes are often clustered in the genome ${ }^{35}$.

The plant defense response is regulated through a complex network of signal transduction pathways involving three signaling molecules: salicylic acid (SA), ethylene and jasmonic acid (JA). Nonexpressor of PR genes 1 (NPR1) and its paralogues, NPR3 and NPR4, are known to function as SA receptors ${ }^{36,37}$. JA is also involved in laticifer cell differentiation, and coronatine insensitive 1 (COI1) has been identified as its receptor ${ }^{38}$. In the H. brasiliensis genome, the COI1, NPR1 and NPR3 genes occur in duplicate pairs and are distributed across different scaffolds (Supplementary Table S10). In contrast, NPR4 is encoded by a single-copy gene. Besides functioning as a defense signaling component, the gaseous phytohormone ethylene is an essential element in regulating rubber tree latex production ${ }^{39}$. The ethylene receptor, ETR1, is encoded by four genes in $H$. brasiliensis. Most of the disease resistance signaling components contain multiple homologues in $\mathrm{H}$. brasiliensis, indicating the importance of these genes in plant defense.

Isoprenoid biosynthesis. Natural rubber from H. brasiliensis is a high molecular weight biopolymer that is made up of cis-isoprene units derived from isopentenyl diphosophate (IPP). The biosynthesis of IPP proceeds via two distinct routes: the cytoplasmic mevalonate pathway (MVA) and the plastidic 2 -C-methyl- ${ }_{\mathrm{D}}$-erythritol 4-phosphate (MEP) pathway (Supplementary Figure S7). In the former, IPP is derived from the condensation of three acetyl-CoA moieties; in the MEP pathway, IPP is formed from the condensation of pyruvate and $\mathrm{D}$-glyceraldehyde 3-phosphate. Radiolabelling of the intermediates of the MVA pathway provided evidence that IPP synthesized from the MVA pathway is utilized for cis-polyisoprene production ${ }^{40,41}$. The MEP pathway has also been considered as a possible alternative means of generating IPP for rubber biosynthesis because the 
expression of 1-deoxy- ${ }^{-}$-xylulose-5-phosphate synthase (DXP synthase) was observed in H. brasiliensis latex and leaves ${ }^{42}$. From our annotation, the $H$. brasiliensis genome has a larger number of MVA and MEP pathway genes than A. thaliana (58 versus 33). For example, DXP synthase, catalyzing the first step in the MEP pathway, has expanded from three genes in A. thaliana to nine in H. brasiliensis. All of the enzymes involved in the MVA and MEP pathways are encoded by multiple genes in H. brasiliensis, indicating the expansion of these gene families in this genome. The expansion of MVA and MEP pathway genes in H. brasiliensis meets the need for its formation of isoprenoids.

Rubber biosynthesis. Latex production is an important biological feature of the rubber tree that has tremendous economic importance. Natural rubber is synthesized by the successive addition of IPP molecules to a priming allylic diphosphate (farnesyl diphosphate and/ or geranylgeranyl diphosphate) ${ }^{43}$. The enzymes leading to the formation of allylic diphosphate from IPP are encoded by multiple genes (isopentenyl-diphosphate delta isomerase, dimethyallyl transferase, farnesyl diphosphate synthase and geranyl geranyl diphosphate synthase) in H. brasiliensis (Supplementary Table S11). The polymerization of cis-1,4-polyisoprene is mediated by a membrane-bound rubber transferase complex with cis-prenyltransferase (CPT) activity ${ }^{44}$. In H. brasiliensis, the entire CPT gene family comprising seven members (designated as CPT1-7) was identified. Based on the phylogenetic analysis of CPT sequences from $H$. brasiliensis and 14 other plant species, five of the seven $H$. brasiliensis CPT genes (CPT1-5) form a subgroup (Fig. 5a). A member from this cluster (CPT2) was shown to exhibit in vitro long-chain prenyltransferase activity in the presence of washed latex particles ${ }^{45}$.

The two most abundant rubber particle proteins, rubber elongation factor (REF) and small rubber particle (SRPP), have been shown to participate in cis-polyisoprene production ${ }^{45}$. In H. brasiliensis, REF and SRPP are encoded by nine and eight genes, respectively, which are the largest numbers reported in any plant genome (Supplementary Figure S8). This significant expansion of rubber biosynthesis-related genes in the H. brasiliensis genome correlates with its capacity to produce high levels of latex. A cis-prenyltransferase-like (CPTL) protein that was suggested to be involved in rubber biosynthesis in lettuce and dandelion ${ }^{46,47}$ was also found in the $H$. brasiliensis genome. Among the annotated rubber biosynthesis-related genes, some are found in gene clusters (Fig. 5b). For example, clusters containing two CPT genes that were likely generated by tandem duplication are distributed along scaffold313 and scaffold548. A larger gene cluster containing seven rubber particle proteins is located on scaffold1741. In addition, SRPP7 and REF8, and SRPP1 and REF6 are arranged in a tandem array on scaffold 1741. Since both gene pairs are phylogenetically close, they may have derived from a tandem duplication event.

CAGE analysis. To understand the transcriptional control of $H$. brasiliensis genes, we analyzed the transcription start sites (TSSs) of genes expressed in different tissues by CAGE analysis. This method enables high-throughput gene expression analysis and the precise mapping of TSSs to the genome ${ }^{48}$. Unique tags that start within the tag cluster are grouped into CAGE-tag starting sites (CTSSs), whereas overlapping CTSSs on the same strand form a tag cluster (TC) ${ }^{49}$. In total, 484 million CAGE tags were sequenced based on libraries prepared from total RNAs isolated from bark, latex and leaf (Supplementary Table S12). Mapping of the CAGE tags to the $H$. brasiliensis genome allowed us to identify 2,948,134 potential TSSs. The CAGE data obtained from biological replicates derived from different tissues showed high correlation, indicating the reproducibility of the analysis (Supplementary Figure S9). The location of the mapped CAGE tags was examined (Supplementary Table S13). In total 31,033 TCs were identified. Approximately 34\% (10,786) of the CAGE tags mapped at most 2,000 bp upstream of the transcripts, allowing for the identification of new TSSs. Accordingly, 54\% $(17,030)$ and $11 \%$ $(3,552)$ of the CAGE tags mapped inside genes or within the intergenic regions, respectively.

Comparative analysis between bark, latex and leaf gene expression data revealed tissue-specific expression related to the function of these tissues. From the 31,033 total TCs identified in H. brasiliensis, 1,136 were found only in latex, 4,158 in bark and 5,516 in leaf (Supplementary Figure S10). GO analysis of the transcripts expressed only in latex revealed an enrichment in genes involved in cellular components including 'cell', 'intracellular', 'organelle' and 'membrane-bounded organelle' categories, which is consistent with the fact that latex is the cytoplasmic content of the laticifer cells (Supplementary Table S14). In bark-specific transcripts, genes belonging to the GO categories 'transcription factor activity, sequence-specific DNA binding', 'catalytic activity' and 'cell wall organization' are enriched (Supplementary Table S15). The enrichment of GO categories 'chloroplast thylakoid membrane', 'pentose-phosphate shunt' and 'photosystem II' that are related to photosynthesis was observed in leaf-specific transcripts (Supplementary Table S16).

CAGE profiling reveals tissue-specific transcription. The expression profiles of rubber biosynthesis-related genes including CPTs, CPTL, SRPPs and REFs in different tissues were analyzed to investigate the genetic basis underlying latex production (Fig. 5c). It was found that CPT1-CP3, CPT5, CPTL, REF1-4, REF6-9, SRPP1-2 and SRPP4-6 were expressed in all the tissues (bark, latex and leaf) examined here (Fig. 6, Supplementary Figures S11-12). Although these key genes associated with rubber biosynthesis were expressed in multiple tissues, the highest expression levels were observed mostly in latex, suggesting the involvement of these genes in long chain cis-polyisoprene production. Their high expression levels most likely correlate with the high latex yield in $H$. brasiliensis. In contrast, CPT4 and CPT6-7 are expressed only in bark and leaf, indicating the possible role of these CPTs in short-chain prenyl product synthesis. Most CPTs, REFs and SRPPs show single dominant peak TSSs except for CPT1, REF1, REF4, REF9, SRPP2, SRPP5 and SRPP8 that have multimodal shapes of TSSs. CAGE-based TSS profiles indicate that some of these rubber biosynthesis-related genes (CPT1, CPT7, REF4, SRPP2 and SRPP8) have different TSSs depending on the tissue. The observation of multiple TSSs from the CAGE analysis provides invaluable resources for identifying alternative promoters that cannot be obtained by other transcriptomic methods. 

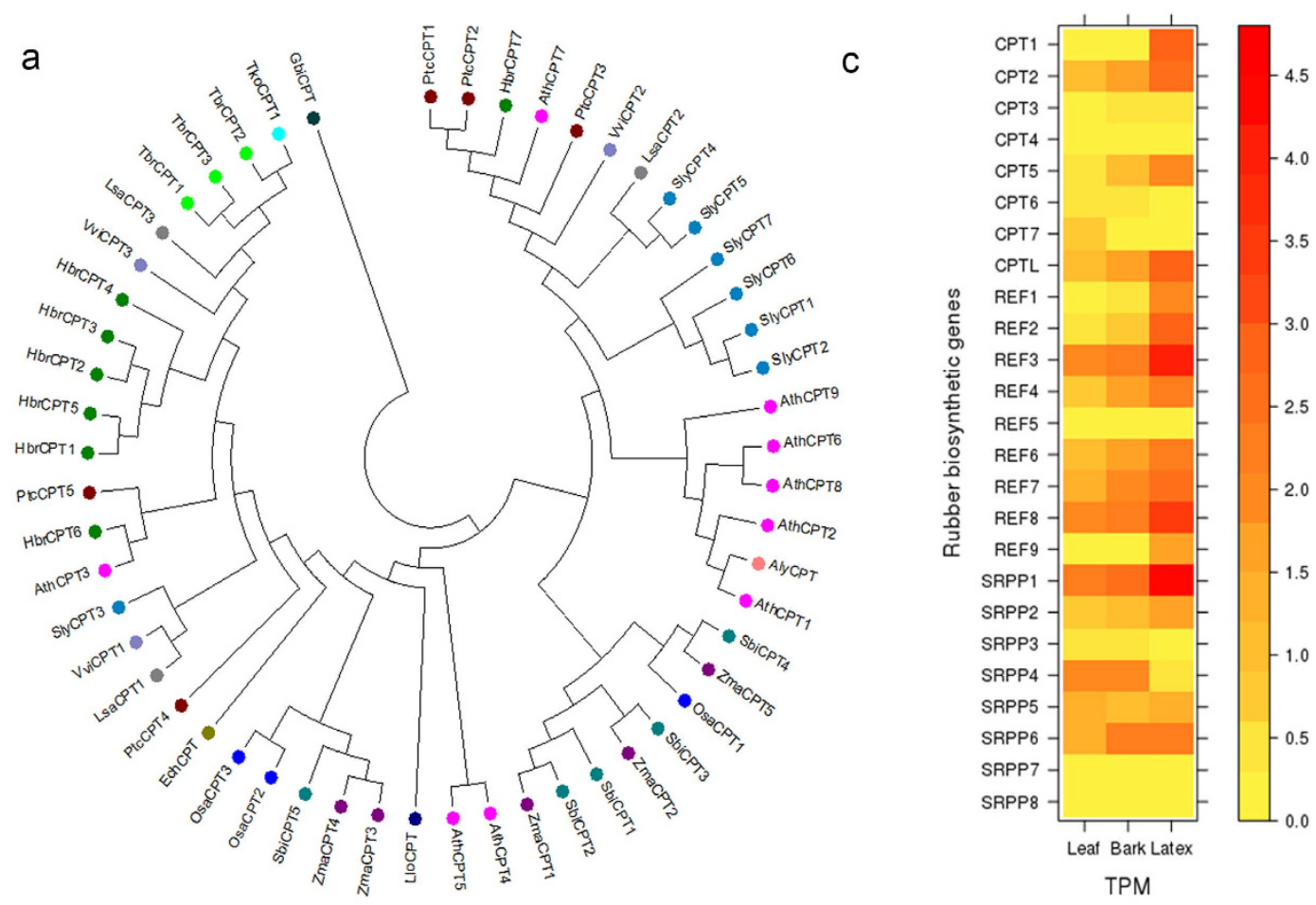

b
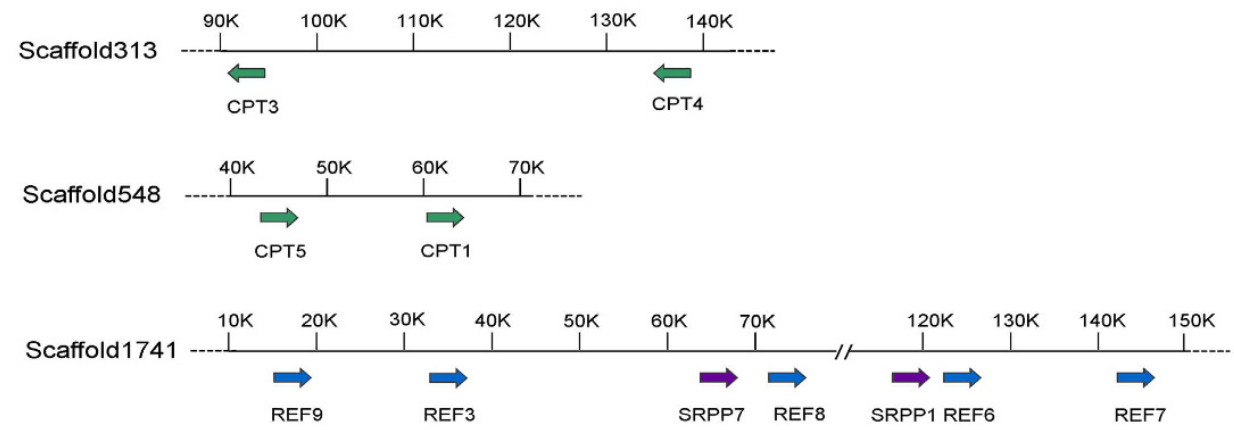

Figure 5. H. brasiliensis rubber biosynthesis-related genes. (a) Phylogenetic analysis of the CPT protein family based on the maximum-likelihood method using MEGA (version 6.06). Three letter codes: Ath, A. thaliana; Aly, Arabidopsis lyrata; Ech, Euphorbia characias; Gbi, Ginkgo biloba; Hbr, H. brasiliensis; Lsa, Lactuca sativa; Llo, Lilium longiflorum; Osa, O. sativa; Ptc, Populus trichocarpa; Sbi, Sorghum bicolor; Sly, S. lycopersicum; Tbr, Taraxacum brevicorniculatum; Tko, Taraxacum kok-saghyz; Vvi, V. vinifera; Zma, Zea mays. (b) Organization of rubber biosynthesis-related gene clusters across the genome. (c) Heat map of the normalized CAGE expression level for genes associated with rubber biosynthesis. The heat map was plotted using the Lattice (version 0.29.33) package in $\mathrm{R}$.

The expression profiles of MVA and MEP pathway genes were also examined to provide insights into the formation of the intermediates associated with rubber biosynthesis. CAGE analysis revealed high expression levels of MVA pathway genes in latex, as compared to leaf and bark (Supplementary Figure S13). One member of the acetyl-CoA acetyltransferase, HMG-CoA synthase and mevalonate diphosphate decarboxylase gene families was highly expressed in latex. In contrast, MEP pathway genes were not preferentially expressed in latex. These observations revealed that the MVA pathway is the main contributor to the formation of the intermediates required for latex production and the enzymes discussed above may be the rate-limiting enzymes for high latex yield.

\section{Discussion}

The H. brasiliensis genome was characterized as high in repeat content and heterozygosity, and with a large genome size that poses technical challenges in assembling its genome. Using a combination of a high coverage Illumina paired-end, mate-pair and long PacBio reads, the H. brasiliensis RRIM 600 genome was assembled. The final assembly spans $1.55 \mathrm{~Gb}$ and represents more than $93.7 \%$ of the transcriptome sequences ${ }^{14,21-24}$ and ESTs from publicly available databases. The current $H$. brasiliensis genome assembly is much improved in continuity, 


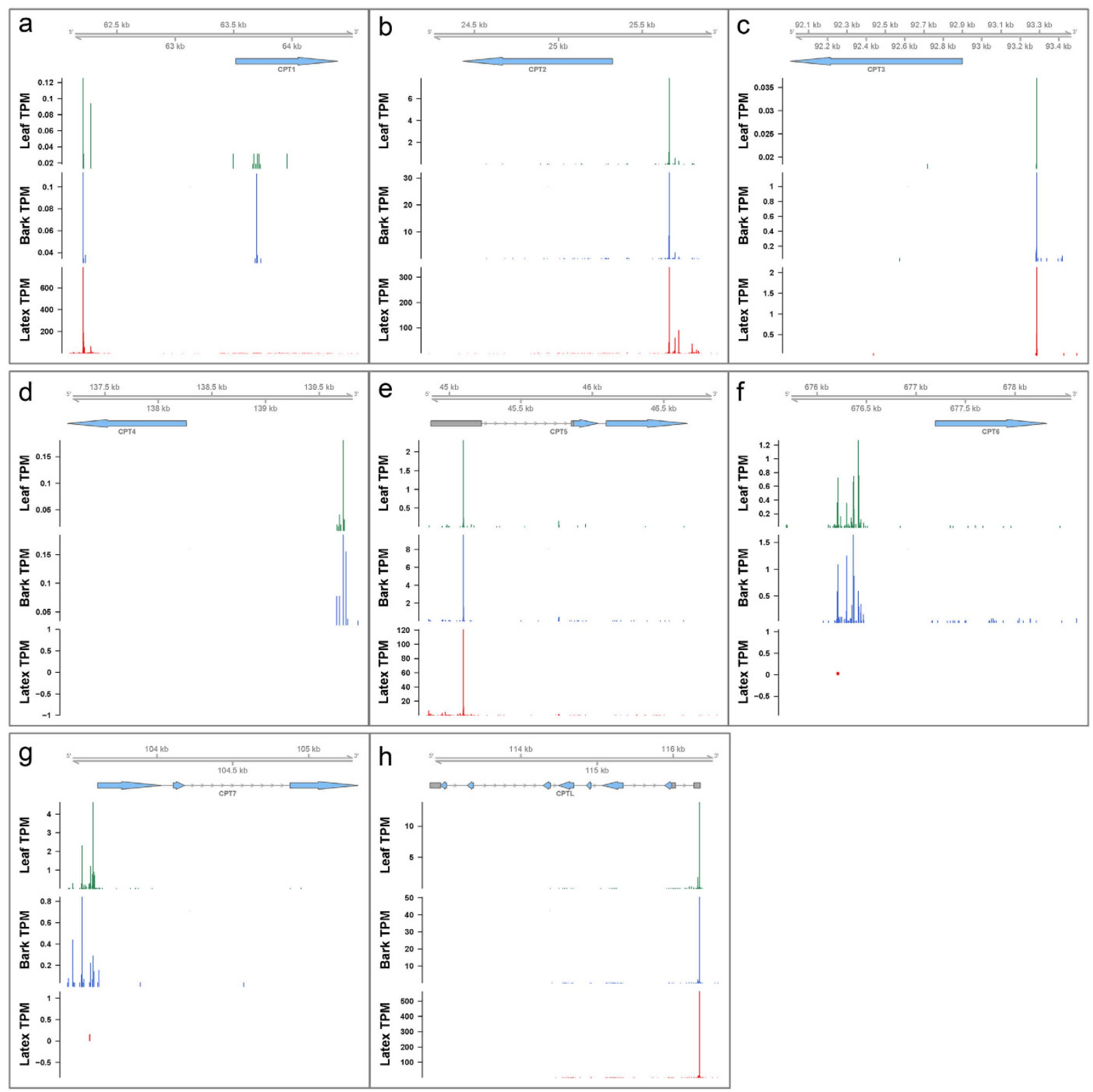

Figure 6. CAGE tag cluster distribution for (a-g) CPT1-7 and (h) CPTL in leaf, bark and latex. The top arrows represent Maker-P predicted gene structures; grey boxes are $5^{\prime} / 3^{\prime}$ UTR and blue arrows are CDSs. The histograms show normalized expression (TPM) and position of TSSs. Each histogram has a different scale for TPM. The relative locations of each gene in the scaffold are indicated at the top of the figure.

allowing for comparative analyses with other plant genomes and genome-wide characterization of protein-coding genes.

Comparative genomics analysis clearly indicates conserved synteny between $\mathrm{H}$. brasiliensis and other members of Euphorbiaceae including M. esculenta, R. communis and J. curcas ${ }^{18-20}$. In most cases, H. brasiliensis gene blocks showed one-to-one, one-to-two and one-to-three synteny relationships with J. curcas, M. esculenta and R. communis, respectively. When compared with other Euphorbiaceae genomes, our analysis revealed that $H$. brasiliensis has the highest number of disease resistance-related genes. The expanded inventory of these genes could conceivably play a role in resistance to abiotic stresses or diseases caused by fungal pathogens. Our genomic and CAGE-seq analyses offer some insights into the biological features that are unique to $\mathrm{H}$. brasiliensis, particularly those that are involved in rubber biosynthesis. The H. brasiliensis genome contains one of the richest set of rubber biosynthesis-related genes including CPTs, REFs and SRPPs. The expansion of these gene families and the high expression of these genes in latex could be an important contributor to high latex production in $H$. brasiliensis. Interestingly, some of these rubber biosynthesis-related genes are organized in clusters in the genome, indicating coordinated evolution and expression for latex production. Expression profiles of rubber biosynthesis-related genes analyzed using CAGE analysis showed that some of these genes exhibit tissue-specific 
TSSs. Although further studies are required to confirm these observations, these results reveal alternative means of transcriptional regulation besides changes in mRNA abundance. Detailed analyses on the expression of these rubber biosynthesis-related genes and their complex transcriptional control provide clues to understanding the unique properties of the rubber tree. The whole-genome sequence information and CAGE-seq data are valuable resources for use in the improvement of the economically important traits of the rubber tree.

\section{Methods}

DNA preparation and genome sequencing. High-quality genomic DNA was extracted from young leaves of $H$. brasiliensis clone RRIM 600 using the Qiagen DNeasy Plant Mini kit (Qiagen, Germany) following the manufacturer's instructions. A 500 bp insert size paired-end library was prepared using the Illumina TruSeq DNA Sample Preparation Kit version 2 (Illumina, USA). Mate-pair libraries with insert sizes of $\sim 3 \mathrm{~Kb}, 5 \mathrm{~Kb}, 7 \mathrm{~Kb}$ and $10 \mathrm{~Kb}$ were prepared according to the Illumina Nextera Mate Pair Sample Prep Kit (Illumina, USA). Sequencing was performed on the Hiseq 2500 and Miseq systems (Illumina, USA). One PacBio library with a $10 \mathrm{~Kb}$ insert size was sequenced with 100 SMRT cells using P5-C3 chemistry. PacBio sequencing was performed on RS II (Pacific Biosciences, USA) at the Yale Center for Genomic Analysis in West Haven, CT, USA.

RNA preparation and CAGE analysis. Total RNAs were isolated from leaf, bark and latex of $H$. brasiliensis clone RRIM 600. Leaf and bark samples were obtained from young rubber plants; latex was collected from mature plants growing at the Centre for Chemical Biology, Penang, Malaysia. Fresh tissues from leaves and bark were flash-frozen in liquid nitrogen and ground to a fine powder. Latex samples were added to the extraction buffer directly. High-quality total RNAs were extracted using the CTAB-LiCl reagent followed by further TRI Reagent ${ }^{\circledR}$ (MRC, USA) treatment ${ }^{50}$. The quality of the purified RNA was confirmed using a Bioanalyzer 2100 (Agilent Technologies, USA). CAGE libraries were prepared according to the protocol described previously ${ }^{51}$ and sequenced on a HiSeq 2500 system. For quality control of the CAGE sequencing data, the TagDust $2^{52}$ program was used to remove data artifacts. For mapping of the CAGE reads to the genome, BWA (version 0.7.12-r1039) ${ }^{53}$ was used. TSS clustering was performed using RECUL (version 1.0) ${ }^{54}$, and finally CAGEr (version 1.10 .3$)^{55}$ was used to analyze the TSSs.

Genome assembly. Raw sequencing reads from each library (Supplementary Table S1) were preprocessed with the program Quake (version 0.3) ${ }^{56}$. Adaptor sequences in the mate-pair reads were trimmed using NextClip

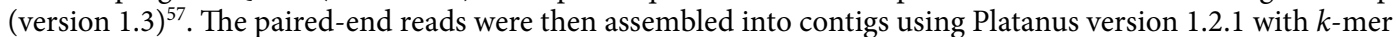
auto-extension $^{15}$. Reads from paired-end and mate-pair libraries were used for scaffolding by increasing library size. Gaps within the scaffolds were filled using the gap-close step in Platanus. Finally, PacBio reads were used to improve the genome assembly with PBJelly version $14.9 .9^{17}$. The PacBio reads were mapped to the assembly using BLASR (version 1.3.1.127046) with parameters: $\operatorname{minMatch}=8$, sdpTupleSize $=8$, minPctIdentity $=75$, bestn $=1$, nCandidates $=10$, maxScore $=500$ and noSplitSubreads. To anchor the assembled scaffolds onto 18 pseudo-chromosomes, 196 RFLP markers from the published genetic map ${ }^{8}$ were mapped to the assembly using BLASTN at an $e$-value cutoff of $1 e-20$. Hits that comprised $>90 \%$ identity and $>85 \%$ coverage were considered mapped markers. Quality of the genome assembly was assessed by mapping all available H. brasiliensis EST sequences downloaded from NCBI and published transcriptome data from leaf, latex and bark tissues ${ }^{14,21-24}$ using BLASTN at a threshold of $90 \%$ identity.

Repeat content. Repeat sequences in the genome assembly were identified using RepeatMasker (version 4.0.5) ${ }^{58}$ with RepBase ${ }^{59}$ and a custom repeat library generated using RepeatModeler (version 1.0.8). RepeatModeler uses Tandem Repeats Finder (version 4.04$)^{60}$ to search for tandem repeats in the genome and two programs, RECON (version 1.08) ${ }^{61}$ and RepeatScout (version 1.0.5) ${ }^{62}$, for de novo identification of repeat families in the genome sequences.

Gene prediction and annotation. Gene models were predicted using the Maker-P pipeline (version $2.31 .8)^{31,32}$. The gene prediction pipeline combined $a b$ initio gene predictions, homology-based searching and transcriptome alignment. Augustus (version 3.0.3) ${ }^{63}$, Snap (version 2013-11-29) , $^{64}$ Fgenesh (version 2.6) ${ }^{65}$ and GeneMark-ES (version 4.21) ${ }^{66}$ were used for de novo gene prediction. Protein sequences from M. esculenta, R. communis, J. curcas, P. trichorpca and the Viridiplantae dataset from Swiss-Prot were used as homology-based evidence. RNA-seq reads from leaf, latex and bark tissues ${ }^{21}$ were assembled using Oases (version 0.2 .8$)^{67}$ and used as transcript evidence. Maker-P was first run using the est2genome $=1$ option to infer gene models directly from transcript evidence. The obtained gene models were then used to train the gene predictors, Augustus and Snap. Maker-P was run iteratively two additional times and the results from one run were used to train gene predictors that were used during the next run. Functional annotation of the predicted gene models was based on comparison with the NCBI, TrEMBL, Swiss-Prot and KEGG databases with a minimal $e$-value of $1 e-5$. GO terms were assigned to the annotated genes using the Blast2GO pipeline (version 3.1.3) ${ }^{68}$. Protein domains and functions were analyzed using InterProScan (version 5.13-52.0) ${ }^{69}$. The tRNAs were identified using tRNAscan-SE (version 1.3.1 $)^{70}$; rRNAs, snRNAs, snoRNAs and microRNAs were identified by searching the genome assembly against the Rfam database (Release 12.0) ${ }^{71}$ using Infernal (version 1.1.1) ${ }^{72}$ with an $e$-value cutoff of $1 e-10$. Transcription factors in $H$. brasiliensis were identified and classified into families using the iTAK pipeline (http://bioinfo.bti. cornell.edu/tool/itak).

Comparative analysis. The non-redundant protein dataset from H. brasiliensis, A. thaliana, O. sativa, $S$. lycopersicum, V. vinifera, M. esculenta, R. communis and J. curcas was used to identify gene clusters. All-against-all BLASTP analysis of all protein sequences was performed with an $e$-value cutoff of $1 e-5$. OrthoMCL (version 2.0.9) ${ }^{73}$ was used to construct gene families with a default inflation value of 1.5. Synonymous $(K s)$ and 
non-synonymous $(\mathrm{Ka})$ substitution rates for orthologous gene pairs in $\mathrm{H}$. brasiliensis and Euphorbiaceae members were computed using the KaKs_Calculator tool (version 2.0$)^{74}$. Syntenic blocks and gene collinearity were inferred using MCScanX (version 2013-11-11) ${ }^{75}$. H. brasiliensis's scaffolds that have more than 15 gene syntenic regions against all four organisms examined (M. esculenta, R. communis, J. curcas and A. thaliana) were visualized using Circos (version 0.67-7).

Identification of disease resistance genes. NBS genes in H. brasiliensis were identified using HMMER (version 3.1 ${ }^{76}$ search analysis to screen the predicted proteome against the raw hidden Markov model (HMM) corresponding to the Pfam NBS (NB-ARC) family domain. The TIR and LRR domains in the predicted NBS-encoding amino acid sequences were screened using HMMER search analysis against the HMM model Pfam TIR and LRR domains, respectively. CC motifs were analyzed using Paircoil2 ${ }^{77}$ with a P-score cutoff of 0.025 .

\section{References}

1. Archer, B. L. \& Audley, B. G. New aspects of rubber biosynthesis. Bot. J. Linn. Soc. 94, 181-196 (1987).

2. Clément-Demange, A., Priyadarshan, P. M., Thuy Hoa, T. T. \& Venkatachalam, P. Hevea rubber breeding and genetics. in Plant Breeding Reviews. 177-283 (John Wiley \& Sons, Inc., 2007).

3. Gonçalves, P.d.S., Cardoso, M. \& Ortolani, A. A. Origin, variability and domestication of Hevea. Pesquisa Agropecuária Brasileira 25, 135-156 (1990).

4. Schultes, R. E. A brief taxonomic view of the genus Hevea, (Malaysian Rubber Research and Development Board, 1990).

5. Priyadarshan, P. M. \& Gonçalves, P.d.S. Use of Hevea gene pool in rubber tree (Hevea brasiliensis Muell. Arg) breeding. The Planter 78, 123-138 (2002).

6. Baldwin, J. J. T. Hevea: A first interpretation. A cytogenetic survey of a controversial genus, with a discussion of its implications to taxonomy and to rubber production. J. Hered. 38, 54-64 (1947).

7. Majumder, S. K. Chromosome studies of some species of Hevea. J. Rubb. Res. Inst. Malaysia 18, 269-273 (1964).

8. Lespinasse, D. et al. A saturated genetic linkage map of rubber tree (Hevea spp.) based on RFLP, AFLP, microsatellite, and isozyme markers. Theor. Appl. Genet. 100, 127-138 (2000).

9. Ong, S. H. Chromosome morphology at the pachytene stage in Hevea brasiliensis: a preliminary report. Proc. Int. Rubb. Conf. 2, 3-12 (1975).

10. Priyadarshan, P. M. Biology of Hevea Rubber, (CABI, Wallingford, UK, 2011).

11. Priyadarshan, P. M. \& Clément-Demange, A. Breeding Hevea rubber: Formal and molecular genetics. in Advances in Genetics, Vol. 52, 51-115 (Academic Press, 2004).

12. d'Auzac, J., Jacob, J.-L. \& Chrestin, H. Physiology of rubber tree latex, (CRC Press, Boca Raton, Florida, 1989).

13. Havanapan, P. O., Bourchookarn, A., Ketterman, A. J. \& Krittanai, C. Comparative proteome analysis of rubber latex serum from pathogenic fungi tolerant and susceptible rubber tree (Hevea brasiliensis). J. Proteomics 131, 82-92 (2016).

14. Rahman, A. Y. et al. Draft genome sequence of the rubber tree Hevea brasiliensis. BMC Genomics 14, 75 (2013).

15. Kajitani, R. et al. Efficient de novo assembly of highly heterozygous genomes from whole-genome shotgun short reads. Genome Res. 24, 1384-1395 (2014).

16. Bennett, M. D. \& Leitch, I. J. Nuclear DNA amounts in angiosperms-583 new estimates. Ann. Bot. 80, 169-196 (1997).

17. English, A. C. et al. Mind the gap: Upgrading genomes with Pacific Biosciences RS long-read sequencing technology. PLoS ONE 7, e47768 (2012).

18. Chan, A. P. et al. Draft genome sequence of the oilseed species Ricinus communis. Nat. Biotech. 28, 951-956 (2010).

19. Sato, S. et al. Sequence analysis of the genome of an oil-bearing tree, Jatropha curcas L. DNA Res. 18, 65-76 (2010).

20. Wang, W. et al. Cassava genome from a wild ancestor to cultivated varieties. Nat. Commun. 5, doi: 10.1038/ncomms6110 (2014).

21. Chow, K.-S., Ghazali, A.-K., Hoh, C.-C. \& Mohd-Zainuddin, Z. RNA sequencing read depth requirement for optimal transcriptome coverage in Hevea brasiliensis. BMC Res. Notes 7, 69 (2014)

22. Li, D., Deng, Z., Qin, B., Liu, X. \& Men, Z. De novo assembly and characterization of bark transcriptome using Illumina sequencing and development of EST-SSR markers in rubber tree (Hevea brasiliensis Muell. Arg.). BMC Genomics 13, 192 (2012).

23. Triwitayakorn, K. et al. Transcriptome sequencing of Hevea brasiliensis for development of microsatellite markers and construction of a genetic linkage map. DNA Research: An International Journal for Rapid Publication of Reports on Genes and Genomes 18, 471-482 (2011).

24. Xia, Z. et al. RNA-Seq analysis and de novo transcriptome assembly of Hevea brasiliensis. Plant Mol. Biol. 77, 299-308 (2011).

25. Noutsos, C., Richly, E. \& Leister, D. Generation and evolutionary fate of insertions of organelle DNA in the nuclear genomes of flowering plants. Genome Res. 15, 616-628 (2005)

26. Sierro, N. et al. The tobacco genome sequence and its comparison with those of tomato and potato. Nat. Commun. 5, doi: 10.1038/ ncomms4833 (2014).

27. Kim, S. et al. Genome sequence of the hot pepper provides insights into the evolution of pungency in Capsicum species. Nat. Genet. 46, 270-278 (2014)

28. Li, F. et al. Genome sequence of the cultivated cotton Gossypium arboreum. Nat. Genet. 46, 567-572 (2014).

29. Paterson, A. H. et al. The Sorghum bicolor genome and the diversification of grasses. Nature 457, 551-556 (2009).

30. Hua-Van, A., Le Rouzic, A., Boutin, T., Filée, J. \& Capy, P. The struggle for life of the genome’s selfish architects. Biology Direct 6, $1-29(2011)$.

31. Campbell, M. S. et al. MAKER-P: a tool kit for the rapid creation, management, and quality control of plant genome annotations. Plant Physiol. 164, 513-524 (2014).

32. Cantarel, B. L. et al. MAKER: An easy-to-use annotation pipeline designed for emerging model organism genomes. Genome Res. 18, $188-196(2008)$

33. Hudson, M., Ringli, C., Boylan, M. T. \& Quail, P. H. The FAR1 locus encodes a novel nuclear protein specific to phytochrome A signaling. Genes Dev. 13, 2017-2027 (1999).

34. Ambawat, S., Sharma, P., Yadav, N. R. \& Yadav, R. C. MYB transcription factor genes as regulators for plant responses: an overview. Physiol. Mol. Biol. Plants 19, 307-321 (2013).

35. Michelmore, R. W. \& Meyers, B. C. Clusters of resistance genes in plants evolve by divergent selection and a birth-and-death process. Genome Res. 8, 1113-1130 (1998).

36. Fu, Z. Q. et al. NPR3 and NPR4 are receptors for the immune signal salicylic acid in plants. Nature 486, 228-232 (2012).

37. Wu, Y. et al. The Arabidopsis NPR1 protein is a receptor for the plant defense hormone salicylic acid. Cell Rep. 1, 639-647 (2012).

38. Tan, D., Sun, X. \& Zhang, J. Age-dependent and jasmonic acid-induced laticifer-cell differentiation in anther callus cultures of rubber tree. Planta 240, 337-344 (2014).

39. Zhu, J. \& Zhang, Z. Ethylene stimulation of latex production in Hevea brasiliensis. Plant Signal Behav. 4, 1072-1074 (2009).

40. Hepper, C. M. \& Audley, B. G. The biosynthesis of rubber from $\beta$-hydroxy- $\beta$-methylglutaryl-coenzyme A in Hevea brasiliensis latex. Biochem. J. 114, 379-386 (1969). 
41. Skilleter, D. N. \& Kekwick, R. G. O. The enzymes forming isopentenyl pyrophosphate from 5-phosphomevalonate (mevalonate 5-phosphate) in the latex of Hevea brasiliensis. Biochem. J. 124, 407-417 (1971).

42. Ko, J. H., Chow, K. S. \& Han, K. H. Transcriptome analysis reveals novel features of the molecular events occurring in the laticifers of Hevea brasiliensis (para rubber tree). Plant Mol. Biol. 53, 479-492 (2003).

43. Cornish, K. Similarities and differences in rubber biochemistry among plant species. Phytochemistry 57, 1123-1134 (2001).

44. Cornish, K. Biochemistry of natural rubber, a vital raw material, emphasizing biosynthetic rate, molecular weight and compartmentalization, in evolutionarily divergent plant species. Nat. Prod. Rep. 18, 182-189 (2001).

45. Asawatreratanakul, K. et al. Molecular cloning, expression and characterization of cDNA encoding cis-prenyltransferases from Hevea brasiliensis. A key factor participating in natural rubber biosynthesis. Eur. J. Biochem. 270, 4671-4680 (2003).

46. Qu, Y. et al. A lettuce (Lactuca sativa) homolog of human Nogo-B receptor interacts with cis-prenyltransferase and is necessary for natural rubber biosynthesis. J. Biol. Chem. 290, 1898-1914 (2015).

47. Epping, J. et al. A rubber transferase activator is necessary for natural rubber biosynthesis in dandelion. Nature Plants 1, 15048 (2015).

48. Shiraki, T. et al. Cap analysis gene expression for high-throughput analysis of transcriptional starting point and identification of promoter usage. Proc. Natl. Acad. Sci 100, 15776-15781 (2003).

49. Carninci, P. et al. Genome-wide analysis of mammalian promoter architecture and evolution. Nat. Genet. 38, 626-635 (2006).

50. Deng, L. H., Luo, M. W., Zhang, C. F. \& Zeng, H. C. Extraction of high-quality RNA from rubber tree leaves. Biosci. Biotechnol. Biochem. 76, 1394-1396 (2012).

51. Takahashi, H., Lassmann, T., Murata, M. \& Carninci, P. $5^{\prime}$ end-centered expression profiling using cap-analysis gene expression and next-generation sequencing. Nat. Protocols 7, 542-561 (2012).

52. Lassmann, T. TagDust2: a generic method to extract reads from sequencing data. BMC Bioinformatics 16, 1-8 (2015).

53. Li, H. \& Durbin, R. Fast and accurate short read alignment with Burrows-Wheeler transform. Bioinformatics 25, 1754-1760 (2009).

54. Ohmiya, H. et al. RECLU: a pipeline to discover reproducible transcriptional start sites and their alternative regulation using capped analysis of gene expression (CAGE). BMC Genomics 15, 269 (2014).

55. Haberle, V., Forrest, A. R. R., Hayashizaki, Y., Carninci, P. \& Lenhard, B. CAGEr: precise TSS data retrieval and high-resolution promoterome mining for integrative analyses. Nucleic Acids Res. 43, e51 (2015).

56. Kelley, D., Schatz, M. \& Salzberg, S. Quake: quality-aware detection and correction of sequencing errors. Genome Biol. 11, R116 (2010).

57. Leggett, R. M., Clavijo, B. J., Clissold, L., Clark, M. D. \& Caccamo, M. NextClip: an analysis and read preparation tool for Nextera Long Mate Pair libraries. Bioinformatics 30, 566-568 (2014).

58. Tarailo-Graovac, M. \& Chen, N. Using RepeatMasker to identify repetitive elements in genomic sequences. Curr. Protoc. Bioinformatics Chapter 4, Unit 4.10 (2009).

59. Jurka, J. Repeats in genomic DNA: mining and meaning. Curr. Opin. Struct. Biol. 8, 333-337 (1998).

60. Benson, G. Tandem repeats finder: a program to analyze DNA sequences. Nucleic Acids Res. 27, 573-580 (1999).

61. Bao, Z. \& Eddy, S. R. Automated de novo identification of repeat sequence families in sequenced genomes. Genome Res. 12, $1269-1276$ (2002).

62. Price, A. L., Jones, N. C. \& Pevzner, P. A. De novo identification of repeat families in large genomes. Bioinformatics 21, i351-i358 (2005).

63. Stanke, M. et al. AUGUSTUS: ab initio prediction of alternative transcripts. Nucleic Acids Res. 34, W435-W439 (2006).

64. Korf, I. Gene finding in novel genomes. BMC Bioinformatics 5, 59 (2004).

65. Salamov, A. A. \& Solovyev, V. V. Ab initio gene finding in Drosophila genomic DNA. Genome Res. 10, 516-522 (2000).

66. Borodovsky, M. \& Lomsadze, A. Eukaryotic gene prediction using GeneMark.hmm-E and GeneMark-ES. Curr. Protoc. Bioinformatics Chapter 4, Unit 4.6.1-10 (2011).

67. Schulz, M. H., Zerbino, D. R., Vingron, M. \& Birney, E. Oases: robust de novo RNA-seq assembly across the dynamic range of expression levels. Bioinformatics 28, 1086-1092 (2012).

68. Conesa, A. et al. Blast2GO: a universal tool for annotation, visualization and analysis in functional genomics research. Bioinformatics 21, 3674-3676 (2005)

69. Quevillon, E. et al. InterProScan: protein domains identifier. Nucleic Acids Res. 33, W116-W120 (2005).

70. Lowe, T. M. \& Eddy, S. R. tRNAscan-SE: A program for improved detection of transfer RNA genes in genomic sequence. Nucleic Acids Res. 25, 955-964 (1997).

71. Griffiths-Jones, S., Bateman, A., Marshall, M., Khanna, A. \& Eddy, S. R. Rfam: an RNA family database. Nucleic Acids Res. 31, 439-441 (2003).

72. Nawrocki, E. P., Kolbe, D. L. \& Eddy, S. R. Infernal 1.0: inference of RNA alignments. Bioinformatics 25, 1335-1337 (2009).

73. Li, L., Stoeckert, C. J. \& Roos, D. S. OrthoMCL: Identification of ortholog groups for eukaryotic genomes. Genome Res. 13, 2178-2189 (2003).

74. Zhang, Z. et al. KaKs_Calculator: calculating Ka and Ks through model selection and model averaging. Genomics Proteomics Bioinformatics 4, 259-263 (2006).

75. Wang, Y. et al. MCScanX: a toolkit for detection and evolutionary analysis of gene synteny and collinearity. Nucleic Acids Res. 40, e49 (2012).

76. Finn, R. D., Clements, J. \& Eddy, S. R. HMMER web server: interactive sequence similarity searching. Nucleic Acids Res. 39, W29-W37 (2011).

77. McDonnell, A. V., Jiang, T., Keating, A. E. \& Berger, B. Paircoil2: improved prediction of coiled coils from sequence. Bioinformatics 22, 356-358 (2006)

\section{Acknowledgements}

This work was conducted under the program of Biomass Engineering Research of RIKEN. This work was also supported by Universiti Sains Malaysia (Rubber Genome grant-1002.PCCB.910206). We thank Prof. Yuichi Hongo, Tokyo Institute of Technology for technical help in mate-pair sequencing.

\section{Author Contributions}

N.-S.L., Y.M., M.K. and M.M. performed genomic analyses. N.-S.L. performed genome assembly and annotation. T.D.T., S.K., A.S.O. and A.C.S.-C. helped to determine the direction of analysis. Y.M and M.K. analyzed CAGE data and constructed GBrowser. N.-S.L. Y.M. and M.M. prepared figures, tables and wrote the manuscript. 


\section{Additional Information}

Accession codes: The H. brasiliensis genome assembly, genome and CAGE sequencing data were deposited at DDBJ/EMBL/GenBank BioProject under accession PRJDB4387. The rubber tree genome database is accessible at http://plant.psc.riken.jp/cgi-bin/gb2/gbrowse/rubber.

Supplementary information accompanies this paper at http://www.nature.com/srep

Competing financial interests: The authors declare no competing financial interests.

How to cite this article: Lau, N.-S. et al. The rubber tree genome shows expansion of gene family associated with rubber biosynthesis. Sci. Rep. 6, 28594; doi: 10.1038/srep28594 (2016).

(c) (i) This work is licensed under a Creative Commons Attribution 4.0 International License. The images or other third party material in this article are included in the article's Creative Commons license, unless indicated otherwise in the credit line; if the material is not included under the Creative Commons license, users will need to obtain permission from the license holder to reproduce the material. To view a copy of this license, visit http://creativecommons.org/licenses/by/4.0/ 